\title{
Genetic Analysis of Walnut Cultivars in China Using Fluorescent Amplified Fragment Length Polymorphism
}

\author{
Qingguo Ma, Junpei Zhang, and Dong Pei ${ }^{1}$ \\ State Key Laboratory of Tree Genetics and Breeding, The Institute of Forestry, The Chinese Academy \\ of Forestry, P.O. Box 1958, Beijing, 100091, P.R. China
}

\begin{abstract}
AdDitional INDEX words. genetic diversity, relationship, Juglans regia
AbstRaCt. Informative DNA fingerprints from 50 walnut cultivars (Juglans regia) in China were generated using amplified fragment length polymorphism (AFLP) markers to reveal their genetic diversity and relationships. Nine primer combinations were selected from $64 \mathrm{EcoR} \mathrm{I} / \mathrm{Mse}$ I primer combinations to amplify the accessions. An average of 132 polymorphic loci per primer set was detected from the nine primer combinations. The discrimination power of each polymorphic marker (estimated by the polymorphism information content) ranged from 0.00 to 0.37 with an average of 0.19 . A moderate level of genetic diversity was observed among the 50 cultivars. Their expected heterozygosity varied from 0.38 to 0.50 (average, 0.44 ), and Dice's similarity coefficient ranged from 0.53 to 0.86 (average, 0.70 ). The cluster analysis conducted using the unweighted pair group method of arithmetic averages method showed that all of the cultivars fell into five groups at Dice's similarity coefficient of 0.68 . According to the comprehensive analyses based on the specific loci, similarity coefficient, and clustering results, six cultivars (Liaoning 1, Zixin, Shanhe 4, Zha 343, Tulare, and Chandler) were considered important germplasms of walnut cultivars.
\end{abstract}

English (or persian) walnut (Juglans regia) is an economically important tree species throughout temperate regions of the world as a result of its nutrient-rich nut and high-quality timber. China is considered one of the most important countries for production of this species and still has wild walnut populations (Kuang and Lu, 1979; Wang et al., 2008). China contributes approximately one-fourth of the world's total walnut production (Food and Agriculture Organization of the United Nations, 2005). In the past 30 years, China has made substantial progress in walnut breeding with more than 40 cultivars developed and used for production. However, as development progressed, distinguishing walnut cultivars became problematic both for purposes of cultivar identification and for defining and protecting intellectual property given that morphological traits are greatly affected by growing season and environmental conditions. Additionally, the lack of information regarding the genetic variability and structure of the available cultivars or elite lines had the potential to limit further genetic improvement. Low genetic similarity among parents limits continued breeding success, whereas the introduction of new sources of germplasms into the breeding pool may provide the genetic variability required to enable continued progress in developing new cultivars (Birchler et al., 2003).

The AFLP technique, developed by Vos et al. (1995), is a polymerase chain reaction-based DNA diversity screening procedure. The sensitivity of the AFLP technique was increased with the introduction of fluorescent markers. It can rapidly generate hundreds of highly repeatable markers from the DNA of

Received for publication 18 Aug. 2011. Accepted for publication 11 Oct. 2011. We gratefully acknowledge financial support from the ' 948 ' Project (No.20094-07) and the key project of the state forestry administration, P.R. China (No.2008-04). The contributions of Meiyong Zhang, Shangfeng Gao, Jing Qi, and Ying Gao are also acknowledged with respect to their assistance on sample collections.

${ }^{1}$ Corresponding author. E-mail: peidonggu@163.com. any organism and is superior in terms of time and cost-efficiency, repeatability, and resolution compared with many other techniques (Aggarwal et al., 2002). Therefore, AFLP markers have emerged as a major genetic marker with broad application in systematics, population genetics, DNA fingerprinting, and mapping quantitative trait loci (Drossou et al., 2004; Mullet et al., 2002; Pan et al., 2002; Vilanova et al., 2003). AFLP analysis has been used to detect DNA polymorphisms and the genetic relationships of walnut genotypes in recent years (Bayazit et al., 2007; Kafkas et al., 2005; Sutyemez, 2006). However, few applications of AFLP technology to the genetic analysis and fingerprinting of walnut cultivars have been reported.

We used the fluorescent AFLP technique to define the genetic variation and structure of $50 \mathrm{~J}$. regia cultivars in China to identify their genetic relationships and the difference of parental genetic contribution as well as to provide molecular information for distinguishing cultivars. The results will provide insight into the genetic background of available cultivars as well as offer scientific guidance on parent selection and breeding in walnut breeding.

\section{Materials and Methods}

Plant materials. In total, 50 cultivars, of which 16 were hybrids (products of controlled pollinations) and 34 were selections (selections from open pollination), were chosen based on either extensive cultivation or predominant characteristics in growth, cropping, and stress resistance, and these cultivars were subjected to AFLP analysis. The breeding of the selected cultivars is briefly described in Table 1. Cultivars were classified according to their most commonly used names. Fresh leaves were collected from 8- to 13-year-old trees in six provinces of P.R. China [Hebei Province, Henan Province, Shanxi Province, Shandong Province, Xinjiang Province, and Yunnan Province (Table 1; Fig. 1)], then frozen quickly and stored at $-80{ }^{\circ} \mathrm{C}$ until DNA extraction. 
Table 1. Accessions chosen for the amplified fragment length polymorphism analysis of walnut cultivars in P.R. China.

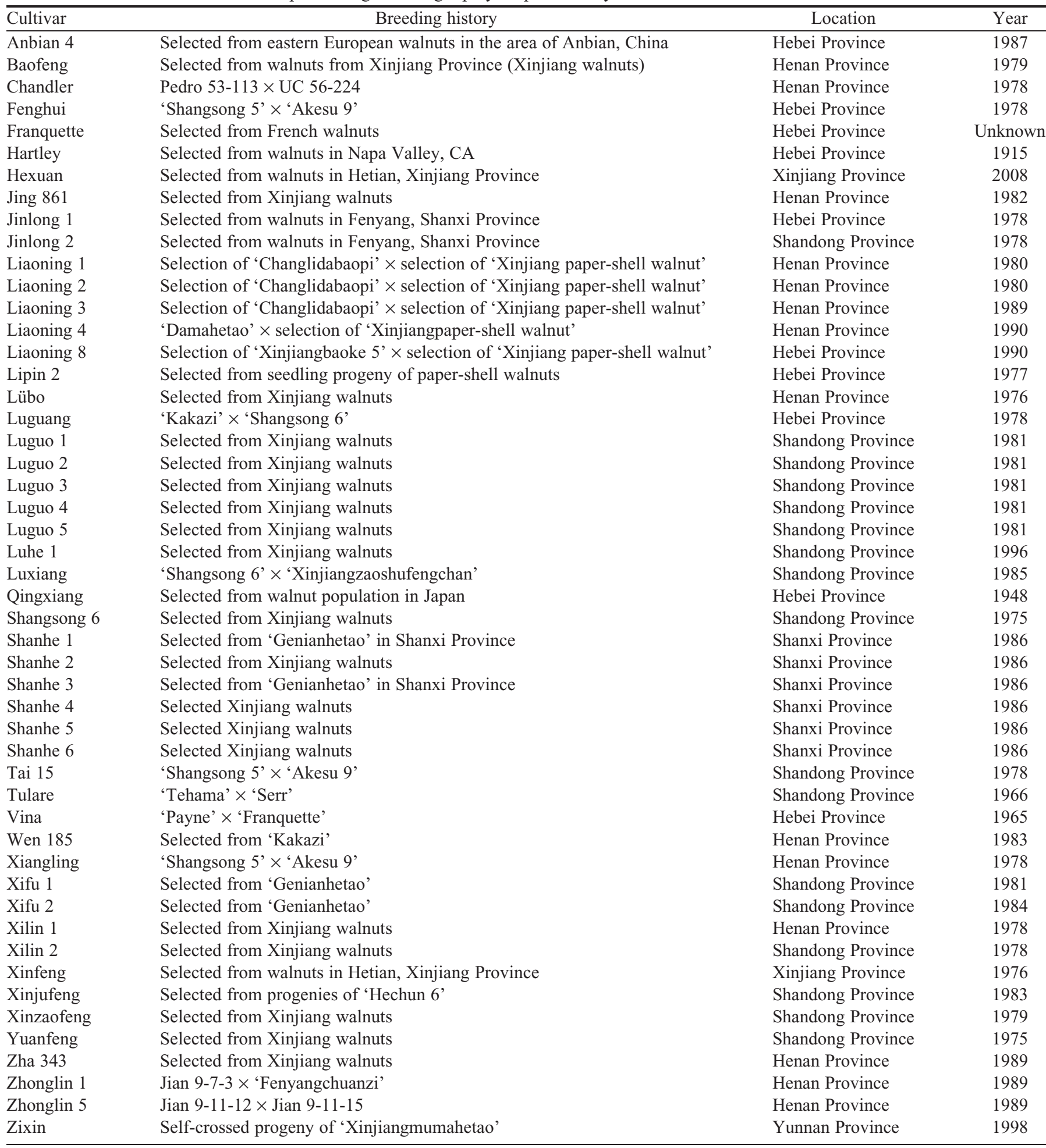

DNA isolation. DNA was extracted from the leaves previously ground in liquid nitrogen. The extraction protocol followed Doyle and Doyle (1990) with minor modifications (Wang et al., 2008). The concentration and intactness of the extracted DNA were determined using a spectrometer at 260 and $280 \mathrm{~nm}$ and by electrophoresis in $0.8 \%$ (w/v) agarose gels against standard solutions of lambda DNA.
Amplified fragment Length POLymorphism ANALYSis. AFLP analysis was carried out according to the manual provided with the AFLP Kit (Dingguo Bio-technology Co., Beijing, China). The primer combinations were fluorescently labeled, and then fragments were detected by laser and accurately sized with an internal standard and with an automated DNA sequencer (ABI PRISM 377; Applied Biosystems, Foster City, CA). Digitally 
converted raw data were saved as samples migrated past the fluorescence detector. Multilocus profiles were visualized using ABI GENESCAN software (Applied Biosystems).

A total of 64 EcoR I/Mse I primer combinations were applied to the genomic DNAs, and nine primer combinations with more polymorphisms were selected to analyze the different accessions of walnut samples (Table 2).

STATistical ANALYSis. Only bands with strong intensity were scored. The AFLP band patterns obtained were classified as present (1) or absent (0) across all 50 walnut cultivars for each primer combination, and the values were used to a compile binary data matrix. Markers with a molecular weight lower than $70 \mathrm{bp}$ were excluded from the data matrix.

The discrimination power of each AFLP marker was evaluated by polymorphism information content [PIC (Anderson et al., 1993)] as follows:

$P I C=1-\sum_{i=1}^{n} P_{i}^{2}-2 \sum_{i=1}^{n} \sum_{j=i+1}^{n} P_{i} P_{j}=2 \sum_{i=1}^{n} \sum_{j=n+1}^{n} P_{i} P_{j}\left(1-P_{i} P_{j}\right)$,

where $n$ is the number of alleles and $P_{i}$ and $P_{j}$ are the frequencies of each allele.

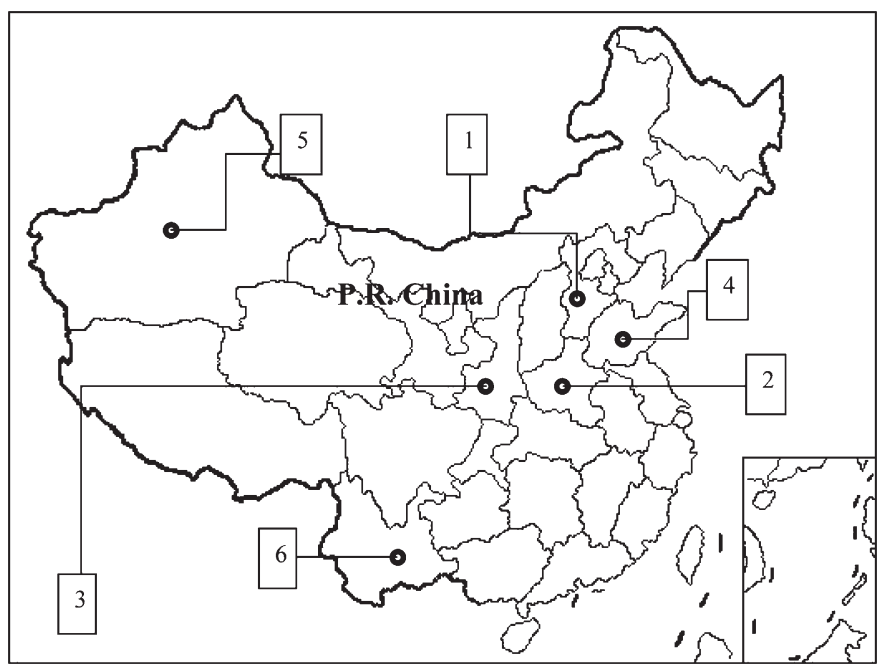

Fig. 1. Names and locations of 50 walnut cultivars sampled in P.R. China; $1=$ Hebei Province, $2=$ Henan Province, $3=$ Shanxi Province, $4=$ Shandong Province, 5 = Xinjiang Province, $6=$ Yunnan Province.

Table 2. Estimates of genetic diversity among 50 walnut cultivars from P.R. China.

\begin{tabular}{lccc}
\hline Primers/indices & $\mathrm{Ne}(\text { mean } \pm \mathrm{SD})^{\mathrm{z}}$ & $\mathrm{I}(\text { mean } \pm \mathrm{SD})^{\mathrm{z}}$ & $\mathrm{He}(\text { mean } \pm \mathrm{SD})^{\mathrm{z}}$ \\
\hline E-AAC/M-CAA & $1.40 \pm 0.33$ & $0.39 \pm 0.23$ & $0.50 \pm 0.37$ \\
E-AAC/M-CTT & $1.34 \pm 0.32$ & $0.34 \pm 0.23$ & $0.39 \pm 0.35$ \\
E-AAG/M-CAA & $1.41 \pm 0.36$ & $0.38 \pm 0.25$ & $0.41 \pm 0.32$ \\
E-AAG/M-CAG & $1.36 \pm 0.35$ & $0.34 \pm 0.25$ & $0.46 \pm 0.37$ \\
E-ACA/M-CAC & $1.39 \pm 0.33$ & $0.37 \pm 0.23$ & $0.45 \pm 0.38$ \\
E-ACT/M-CTA & $1.45 \pm 0.35$ & $0.41 \pm 0.23$ & $0.38 \pm 0.31$ \\
E-AGC/M-CAG & $1.45 \pm 0.35$ & $0.41 \pm 0.23$ & $0.46 \pm 0.34$ \\
E-AGC/M-CAT & $1.41 \pm 0.35$ & $0.37 \pm 0.26$ & $0.50 \pm 0.37$ \\
E-AGC/M-CTT & $1.37 \pm 0.32$ & $0.37 \pm 0.23$ & $0.40 \pm 0.33$ \\
Average & $1.39 \pm 0.03$ & $0.37 \pm 0.03$ & $0.44 \pm 0.05$
\end{tabular}

$\overline{\mathrm{z}} \mathrm{Ne}=$ effective number of alleles; I = Shannon' $\mathrm{s}$ information index; $\mathrm{He}=$ expected heterozygosity.
A similarity matrix was calculated with the Dice's similarity coefficient (Dice, 1945), and cluster analysis was carried out using unweighted pair group method of arithmetic averages [UPGMA (Gil-Vega et al., 2006)] with NTSYSpc 2.11F software package (Rohlf, 1998).

Factorial correspondence analysis (FCA) was performed with GENETIX 4.05 software (Belkhir et al., 2000; Breton et al., 2008).

The percentage of polymorphic bands was calculated using POPGENE32 Version 1.32 (Yeh et al., 1997), which was also used to carry out the effective number of alleles $(\mathrm{Ne})$ and Shannon's information index (I) on the premise of the HardyWeinberg equilibrium. The expected heterozygosity $(\mathrm{He})$ index was estimated according to Nei (1987) as

$$
H e=1-\sum_{i=1}^{n} P_{i}^{2},
$$

where $P i$ is the frequency of the $i$ th allele scored as 0 (band absent).

\section{Results}

AMPLIFIEd FRAGMENT LENGTH POLYMORPHISMS. Nine primer combinations produced clear-cut AFLP profiles for the 50 walnut cultivars, and a total of 1263 AFLP fragments were detected. The fragment size scored in all accessions ranged from 70 to $500 \mathrm{bp}$ with the majority distributed between 80 and $300 \mathrm{bp}$. A total of 1189 fragments among the total 1263 tested exhibited polymorphisms. The number of fragments per primer combination ranged from $116(E-\mathrm{ACA} / M$-CAC) to $171(E-$ $\mathrm{AAG} / M$-CAA) with an average of 140 , whereas polymorphic fragments varied from 110 (E-ACA/M-CAC) to 160 (E-AAG/ $M$-CAA) with an average of 132 , and the percentage of polymorphism ranged from $88.7 \%$ to $99.3 \%$ with an average of $94.1 \%$. Eight of the nine primer combinations generated greater than $90 \%$ of the polymorphisms detected.

Genetic Diversity. Three indexes ( $\mathrm{Ne}, \mathrm{I}$, and $\mathrm{He}$ ) were calculated based on the allele frequencies in nine primer combinations (Table 2). Ne varied from 1.00 to 2.00 with an average of 1.39. I changed from 0.00 to 0.69 with an average of 0.37 . He of each allele ranged from 0.00 to 1.00 with an average of 0.44 .

Cultivar discrimination. The frequency of polymorphic bands observed among the 50 cultivars ranged from 0.14 to 0.99 with an average of 0.67 . The discrimination power of each marker was estimated by the PIC (results not shown) and ranged between 0.00 and 0.37 (the expected maximum value for a biallelic locus was 0.50 ) with an average of $0.19 . E-\mathrm{ACT} / M$-CTA showed the highest PIC value of 0.37. A large proportion of markers had a high discrimination power above 0.30 .

The discrimination power of the nine primer combinations was estimated by computing the number of possible groups into which the 50 cultivars could be separated. Examination of all loci revealed that the 50 cultivars could be distinguished by each of the nine primer combinations, and the identification percentage per primer combination was $100 \%$.

The Dice's similarity coefficient among cultivars ranged from 0.53 to 0.86 with an average of 0.70 .

GENETIC STRUCTURE AND RELATIONSHIPS. FCA calculated on cultivar polymorphism AFLP data revealed that the first axis (axis 1) explained $8.40 \%$ of the percentage variation when the third axis (Axis 3) explained 5.87\% and it also revealed two 
main groups of cultivars comprising 39 individuals, whereas the remaining 11 cultivars were dispersed (Fig. 2). Group 1 included 21 walnut cultivars collected from Shanxi Province, Shandong Province, and Xinjiang Province, whereas Group 2 comprised 18 cultivars collected from Hebei Province and Henan Province. Interestingly, 'Shanhe 2' and 'Shanhe 4' were clustered together but isolated from all others as were 'Baofeng' and 'Xinzaofeng'. 'Liaoning 1', 'Franquette', 'Zha343', 'Tai 15 ', and 'Zixin' were dispersed. 'Chandler' and 'Tulare' were also separated in the FCA.

A dendrogram was generated from the data with the Dice index, UPGMA, and the program NTSYSpc 2.10t (Fig. 3). No ties were encountered during the UPGMA analysis.

The dendrogram indicated that the 50 cultivars were divided into five groups (I to V) at a level of 0.68 (the mean genetic similarity coefficient investigated) (Fig. 3). Group I contained the cultivar Liaoning 1; Group III consisted of 'Zha 343'; Group V had the cultivars Tulare and Chandler; Group II contained 24 cultivars; and Group IV was comprised of 22 cultivars (Fig. 3).

Regardless of the estimator applied, 'Luguo 1' and 'Luguo 3 ' were the most closely related cultivars with a Dice similarity of 0.8587. 'Liaoning 1' and 'Chandler' had the lowest genetic similarity with a Dice similarity of 0.5318 .

The lowest genetic distances were observed among closely related cultivars (Fig. 3). 'Liaoning 2' and 'Liaoning 8' were highly similar to the 'Xinjiang paper-shell walnut' (with a Dice similarity of 0.8145 ), whereas 'Zhonglin 5', 'Xiangling', and 'Luguang' shared origins with walnuts from Xinjiang Province (hereafter referred to as Xinjiang walnuts). 'Vina' was generated from 'Franquette' (with a Dice similarity of 0.8310 ), and 'Baofeng' and 'Zhonglin 1' were generated from Xinjiang walnuts (with a Dice similarity of 0.8090 ). 'Luguo 1', 'Luguo 3', and 'Luguo 4' were derived from a seedling progeny of Xinjiang walnuts in Shandong, and 'Xinjufeng', 'Luguo 2', and 'Luxiang' shared close relatedness with Xinjiang walnuts such that these six cultivars were clustered. 'Hexuan' and 'Xinfeng' were both selected from Xinjiang walnuts in Hetian County (with a Dice similarity of 0.8321 ), whereas 'Shanhe 1' and 'Shanhe 3' were derived from 'Genianhetao' (with a Dice similarity of 0.8118 ). 'Shanhe 2', 'Shanhe 4', 'Shanhe 5', and 'Shanhe 6' were selected from seedling progenies of Xinjiang walnuts in Shanxi Province.

The genetic similarity coefficient and dendrogram among 'Shangsong 6' and its progenies 'Luxiang' (with a Dice similarity of 0.8301 ) and 'Luguang' (with a Dice similarity of 0.6413 ) indicated a higher genetic similarity with the female parent. The dendrogram showed a degree of variation among full-sib families ('Liaoning 1' and 'Liaoning 3'; 'Fenghui', 'Xiangling', and 'Tai 15').

\section{Discussion}

A variety of phenotypic, biochemical, and molecular markers have previously been used for the genetic characterization of walnut genotypes, including phenotypic markers (Zeneli et al., 2005), isozymes (Fornari et al., 2001; Vyas et al., 2003), randomly

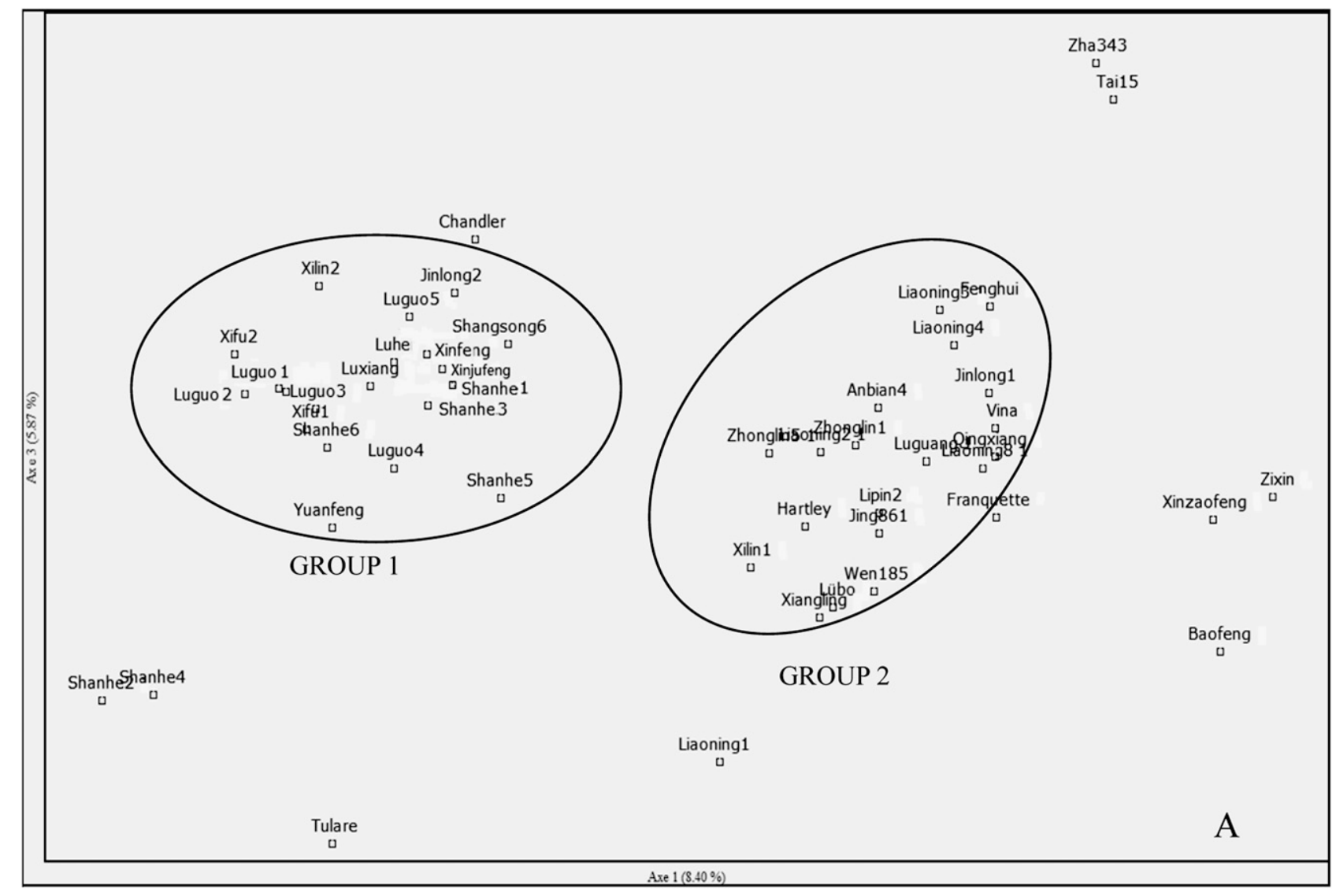

Fig. 2. Factorial correspondence analysis (FCA) based on polymorphisms at amplified fragment length polymorphism loci for 50 cultivars from P.R. China chosen to represent the molecular diversity of walnuts. 


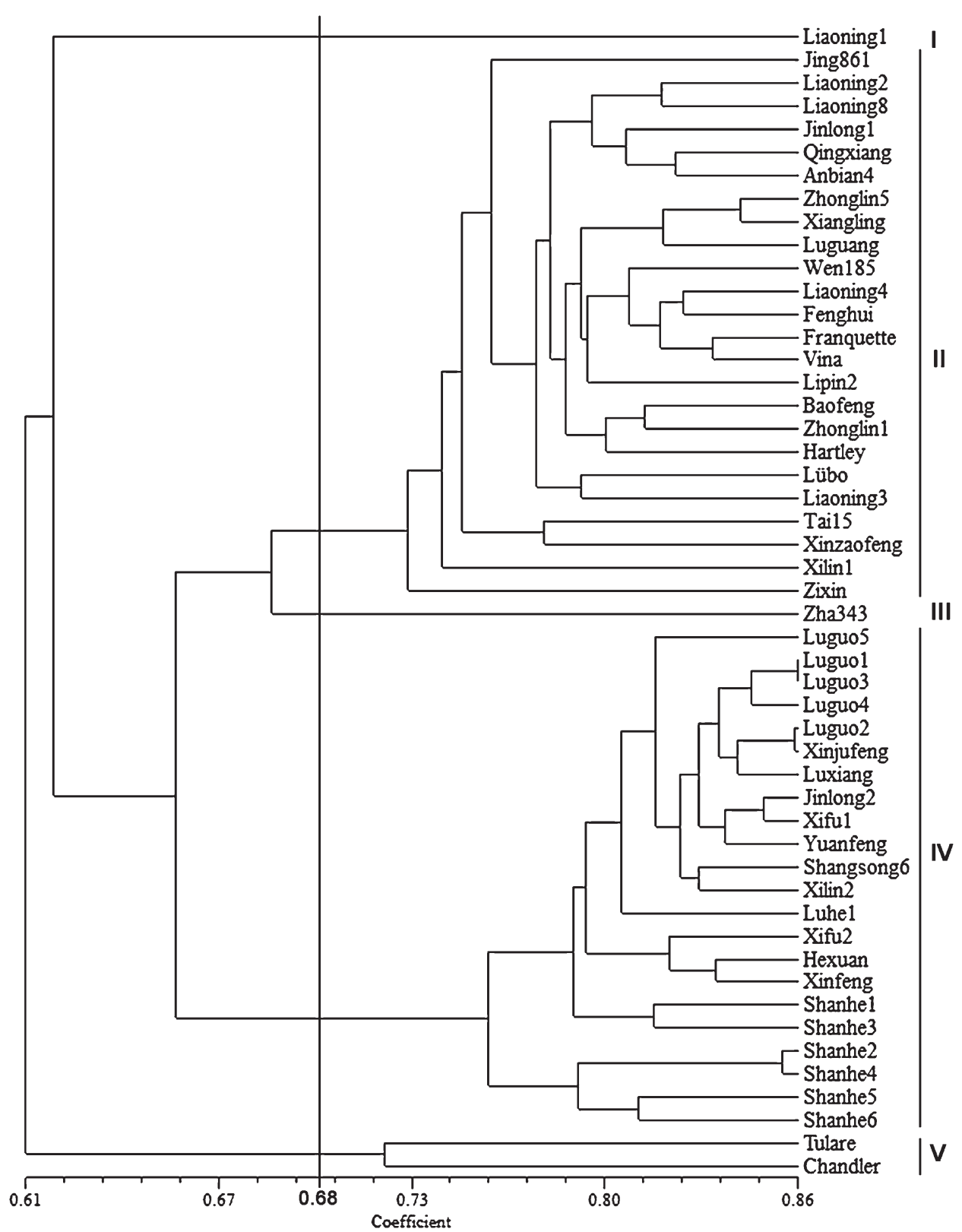

Fig. 3. Dendrogram of 50 walnut cultivars from P.R. China based on amplified fragment length polymorphism analysis conducted using nine primer combinations.

amplified polymorphic repeats (RAPD) (Nicese et al., 1998), restriction fragment length polymorphisms (RFLPs) (Fjellstrom and Parfitt, 1994), intersimple sequence repeats (ISSRs) (Potter et al., 2002), simple sequence repeats (SSRs) (Dangl et al., 2005; Foroni et al., 2005; Victory et al., 2006; Wang et al., 2008), and AFLP (Bayazit et al., 2007). Here, AFLP markers were successfully used for the identification and genetic analysis of walnut cultivars. The results were repeatable, precise, and robust, and the data revealed a high level of polymorphisms among walnut cultivars with an average polymorphism rate of $94.1 \%$ (88.7\% to $99.3 \%)$. This was relatively high compared with that estimated using other marker systems such as RAPD [25\% in walnut (Nicese et al., 1998)], ISSR [51.52\% in Arecaceae (Roncal et al., 2007)], SSR [83.33\% in Psathyrostachys huashanica (Wang et al., 2006a)]. It was comparable to those in Boesenbergia
[98.7\% to $100 \%$ (Techaprasan et al., 2008)], Erythroxylum [94.4\% (Johnson et al., 2005)], Amaranthus [94\% to $99 \%(\mathrm{Xu}$ and Sun, 2001)], Soldanella [98.38\% to $99.29 \%$ (Zhang et al., 2001)] but higher than those in walnut $50 \%$ (Bayazit et al., 2007), 28.4\% (Sutyemez, 2006)]. The technique was demonstrated to be effective for discriminating genotypes, defining genetic structure, and assessing genetic relationships among walnut cultivars. The discrimination power of each polymorphic marker (estimated by the PIC) ranged between 0.00 and 0.38 with an average of 0.19 . The most polymorphic primer combination revealed 160 polymorphic markers with an average of 132 per primer combination, suggesting that the AFLP technique was very efficient for cultivar identification. It was previously reported that AFLP technology could distinguish among cultivars in which no difference was apparent on the basis of RFLP data (Lombard et al., 2000). Here, all of the 50 cultivars could be effectively distinguished using only one primer combination.

The dendrogram assigned five groups (I to $\mathrm{V}$ ) among the cultivars with the SC level set at 0.68 (Fig. 3). Groups I and III contained only one cultivar each, Liaoning 1 and Zha 343, respectively. Group V included two cultivars (Tulare and Chandler), and Groups II and IV comprised 24 and 22 cultivars, respectively. Cultivars in Group IV showed a common original background; they were developed from hybrids or selections of Xinjiang walnuts with the exception of 'Jinlong 2', which was selected from progenies of 'Fenyanghetao', and 'Xifu 1', 'Xifu 2', 'Shanhe 1', and 'Shanhe 3', which were selected from progenies of 'Genianhetao'. Additionally, all of the Group IV cultivars except 'Jinlong 2' were precocious types. Group II showed more diversity in terms of cultivar origin, including both hybrids and natural selections, both precocious and nonprecocious maturing types and both Chinese local cultivars and introduced non-Chinese cultivars. The FCA showed a similar clustering result to the dendrogram with two main groups. Specifically, cultivars collected from Shandong Province, Shanxi Province, and Xinjiang Province were clustered [Group 1 (Fig. $2)$ ] as were those from Henan Province and Hebei Province [Group 2 (Fig. 2)]. However, several cultivars from these six provinces were also dispersed. This could reflect their complex selection history.

The results of this study suggest moderate genetic diversity among walnut cultivars in China compared with other tree species 
(Wang et al., 2008) with their D varying between 0.64 and 0.83 . Among the 50 cultivars examined, some important germplasms were identified according to their similarity coefficients or their specific loci. 'Liaoning 1', 'Zixin', 'Shanhe 4', 'Zha 343', 'Tulare', and 'Chandler' varied substantially from the other cultivars with similarity coefficients less than 0.68. Furthermore, the specific loci on which only one single cultivar generated a band among 'Liaoning 1', 'Zixin', 'Tulare', and 'Chandler' ranged from 10 to 28 . 'Zha 343' was a selection of Xinjiang walnuts, whereas 'Zixin' was derived from the self-crossed progeny of 'Xingjiang Muma' in Yunan Province. 'Chandler' and 'Tulare' are introduced elite cultivars from the United States. 'Liaoning 1' has the same parent as 'Liaoning 2' and 'Liaoning 3', but they were grouped apart, which remains unexplained but may suggest that relatively dramatic genetic events took place during the breeding process of 'Liaoning 1' compared with 'Liaoning 2' and 'Liaoning 3'.

Notably, cultivars with the same genetic origin were not assigned to the same groups. For example, 'Fenghui', 'Xiangling', and 'Tai 15' were hybrids that shared the same parents as 'Shangsong 5' and 'Akesu 9'; however, these cultivars did not cluster together and were instead dispersed among several subgroups. These results may be indicated that dominant AFLP markers cannot make pure clusters or that the level of diversity selected for separating groups fell within the range observed within a segregating family generated by hybridizing two highly heterozygous parents. Similar results were obtained for Ginkgo biloba (Wang et al., 2006b).

'Luguo 1', 'Luguo 3', 'Luguo 2', and 'Xinjufeng' were identified as the most closely related cultivars. These were selected from Xinjiang walnuts, although they differ morphologically (Xi and Zhang, 1996; Zhang et al., 2008), suggesting that they exhibit relatively less genetic variation or that they might be derived from a mutant or that they may be segregating for the morphological trait or traits.

The AFLP profiles and the clusters established here can be used for germplasm identification. Additional work will be required to determine if the fingerprint, genetic diversity, and similarity information can provide molecular solutions for parent selection in cross-breeding and effective marker-assisted genetic improvement.

\section{Literature Cited}

Aggarwal, R.K., V.V. Shenoy, J. Ramadevi, R. Rajkumar, and L. Singh. 2002. Molecular characterization of some Indian Basmati and other elite rice genotypes using fluorescent-AFLP. Theor. Appl. Genet. 105:680-690.

Anderson, J.A., G.A. Churchill, J.E. Autrique, S.D. Tanksley, and M.E. Sorrells. 1993. Optimizing parental selection for genetic linkage maps. Genome 36:181-186.

Bayazit, S., K. Kazan, S. Gülbitti, V. Çevik, H. Ayanoğlu, and A. Ergül. 2007. AFLP analysis of genetic diversity in low chill requiring walnut (Juglans regia L.) genotypes from Hatay, Turkey. Sci. Hort. 111:394-398.

Belkhir, K., V. Castric, and F. Bonhomme. 2000. GENETIX 4.05, logiciel sous Windows ${ }^{\mathrm{TM}}$ pour la génétique des populations. 1 Mar. 2011. <http://www.univ-montp2.fr/genetix/identix_ms.pdf >.

Birchler, J.A., D.L. Auger, and N.C. Riddle. 2003. In search of the molecular basis of heterosis. Plant Cell 15:2236-2239.

Breton, C., C. Pinatel, F. Médail, F. Bonhomme, and A. Bervillé. 2008. Comparison between classical and Bayesian methods to investigate the history of olive cultivars using SSR-polymorphisms. Plant Sci. 175:524-532.
Dangl, G.S., K. Woeste, M.K. Aradhya, A. Koehmstedt, C. Simon, D. Potter, C. Leslie, and G.H. McGranahan. 2005. Characterization of 14 microsatellite markers for genetic analysis and cultivar identification of walnut. J. Amer. Soc. Hort. Sci. 130:348-354.

Dice, L.R. 1945. Measures of the amount of ecologic association between species. Ecology 26:297-302.

Doyle, J.J. and J.L. Doyle. 1990. Isolation of plant DNA from fresh tissue. Focus 12:13-15.

Drossou, A., A. Katsiotis, J.M. Leggett, M. Loukas, and S. Tsakas, 2004. Genome and species relationships in genus Avena based on RAPD and AFLP molecular markers. Theor. Appl. Genet. 109:4854.

Fjellstrom, R.G. and D.E. Parfitt. 1994. RFLP inheritance and linkage in walnut. Theor. Appl. Genet. 89:665-670.

Food and Agriculture Organization of the United Nations. 2005. Agricultural production, crops primary, Geneva. 13 Nov. 2006. $<$ http://apps.fao.org/faostat/>.

Fornari, B., M.E. Malvolti, D. Taurchini, S. Fineschi, I. Beritognolo, E. Maccaglia, and F. Cannata. 2001. Isozyme and organellar DNA analysis of genetic diversity in natural/naturalised European and Asiatic walnut (Juglans regia L.) populations. Acta Hort. 544:167178.

Foroni, I., R. Rao, K. Woeste, and M. Gallitelli. 2005. Characterisation of Juglans regia L. with SSR markers and evaluation of genetic relationships among cultivars and the 'Sorrento' landrace. J. Hort. Sci. Biotechnol. 80:49-53.

Gil-Vega, K., C. Díaz, A. Nava-Cedillo, and J. Simpson. 2006. AFLP analysis of Agave tequilana varieties. Plant Sci. 170:904-909.

Johnson, E.L., D. Zhang, and S.D. Emche. 2005. Inter- and intraspecific variation among five Erythroxylum taxa assessed by AFLP. Ann. Bot. (Lond.) 95:601-608.

Kafkas, S., H. Ozkan, and M. Sutyemez. 2005. DNA polymorphism and assessment of genetic relationships in walnut genotypes based on AFLP and SAMPL markers. J. Amer. Soc. Hort. Sci. 130:585-590.

Kuang, K.Z. and A.M. Lu. 1979. Flora of China. 21 [in Chinese]. Science Press, Beijing, China.

Lombard, V., C.P. Baril, P. Dubreuil, F. Blouet, and D. Zhang. 2000. Genetic relationships and fingerprinting of rapeseed cultivars by AFLP. Crop Sci. 40:1417-1425.

Mullet, J.E., R.R. Klein, and P.E. Klein. 2002. Sorghum bicolor-An important species for comparative grass genomics and a source of beneficial genes for agriculture. Curr. Opin. Plant Biol. 5:118-121.

Nei, M. 1987. Molecular evolutionary genetics. Columbia University Press, New York, NY.

Nicese, F.P., J.I. Hormaza, and G.H. McGranahan. 1998. Molecular characterization and genetic relatedness among walnut (Juglans regia L.) genotypes based on RAPD markers. Euphytica 101:199206.

Pan, Z., S. Kawabata, N. Sugiyama, R. Sakiyama, and Y. Cao. 2002. Genetic diversity of cultivated resources of pear in north China. Acta Hort. 587:187-194.

Potter, D., F. Gao, G. Aiello, C. Leslie, and G.H. McGranahan. 2002. Inter simple sequence repeat markers for fingerprinting and determining genetic relationships of walnut (Juglans regia) cultivars. J. Amer. Soc. Hort. Sci. 127:75-81.

Rohlf, F.J. 1998. NTSYS-pc, Numerical taxonomy and multivariate analysis system. Version 2.11F. Exeter Publications, Setauket, NY.

Roncal, J., J. Francisco-Ortega, and C.E. Lewis. 2007. An evaluation of the taxonomic distinctness of two Geonoma macrostachys (Arecaceae) varieties based on intersimple sequence repeat (ISSR) variation. Bot. J. Linn. Soc. 153:381-392.

Sutyemez, M. 2006. Comparison of AFLP polymorphism in progeny derived from dichogamous and homogamous walnut genotypes. Pak. J. Biol. Sci. 9:2303-2307.

Techaprasan, J., S. Klinbunga, and T. Jenjittikul. 2008. Genetic relationships and species authentication of Boesenbergia (Zingiberaceae) in Thailand based on AFLP and SSCP analyses. Biochem. Syst. Ecol. 36:408-416. 
Victory, E.R., J.C. Glaubitz, O.E. Rhodes, Jr., and K.E. Woeste. 2006. Genetic homogeneity in Juglans nigra (Juglandaceae) at nuclear microsatellites. Amer. J. Bot. 93:118-126.

Vilanova, S., C. Romero, A.G. Abbott, G. Llacer, and M.L. Badenes. 2003. An apricot (Prunus armeniaca L.) F2 progeny linkage map based on SSR and AFLP makers, mapping plum pox virus resistance and self-incompatibility traits. Theor. Appl. Genet. 107:239247.

Vos, P., R. Hogers, M. Bleeker, M. Reians, T. Lee, M. Hornes, A. Frijters, J. Pot, J. Peleman, J. Peleman, M. Kuiper, and M. Zabeau. 1995. AFLP: A new technique for DNA fingerprinting. Nucleic Acids Res. 23:4407-4414.

Vyas, D., S.K. Sharma, and D.R. Sharma. 2003. Genetic structure of walnut genotype using leaf isozymes as variability measure. Sci. Hort. 97:141-152.

Wang, H., D. Pei, R.S. Gu, and B.Q. Wang. 2008. Genetic diversity and structure of walnut populations in central and southwestern China. J. Amer. Soc. Hort. Sci. 133:197-203.

Wang, L., J. Guo, and G.F. Zhao. 2006a. Genetic diversity of the endangered and endemic species Psathyrostachys huashanica natural populations using simple sequence repeats (SSRs) markers. Biochem. Syst. Ecol. 34:310-318.
Wang, L., S.Y. Xing, K.Q. Yang, Z.H. Wang, Y.Y. Guo, and H.R. Shu. 2006b. Genetic relationships of ornamental cultivars of Ginkgo biloba analyzed by AFLP techniques. Acta Genetica Sinica 33:1020-1026.

Xi, R.T. and Y.P. Zhang. 1996. Fruit trees of China. Walnut [in Chinese]. Chinese Forestry Press, Beijing, China.

Xu, F. and M. Sun. 2001. Comparative analysis of phylogenetic relationships of grain Amaranths and their wild relatives (Amaranthus, Amaranthaceae) using internal transcribed spacer, amplified fragment length polymorphism, and double-primer fluorescent intersimple sequence repeat markers. Mol. Phylogenet. 21:372-387.

Yeh, F., Q.Y. Yang, and T. Boyle. 1997. POPGENE. Microsoft Windows-based freeware for population genetic analysis. Release 1.32. University of Alberta, Edmonton, AB, Canada.

Zeneli, G., H. Kola, and M. Dida. 2005. Phenotypic variation in native walnut populations of northern Albania. Sci. Hort. 105:91-100.

Zhang, L.B., H.P. Comes, and J.W. Kadereit. 2001. Phylogeny and quaternary history of the European montane/alpine endemic Soldanella (Primulaceae) based on ITS and AFLP variation. Amer. J. Bot. 88:2331-2345.

Zhang, M.Y., Y. Xu, L. Xu, X. Wang, and L. Gao. 2008. Walnut cultivars suitable for cultivation in Shandong Province [in Chinese]. Deciduous Fruits 1:11-13. 